\title{
Energy localization invariance of tidal work in general relativity
}

\author{
Marc Favata* \\ Theoretical Astrophysics, California Institute of Technology, Pasadena, California 91125
}

(Received 24 August 2000; published 13 February 2001)

\begin{abstract}
It is well known that when an external general relativistic (electric-type) tidal field $\mathcal{E}_{j k}(t)$ interacts with the evolving quadrupole moment $\mathcal{I}_{j k}(t)$ of an isolated body the tidal field does work on the body ("tidal work") — i.e., it transfers energy to the body—at a rate given by the same formula as in Newtonian theory: $d W / d t=$ $-\frac{1}{2} \mathcal{E}_{j k} d \mathcal{I}_{j k} / d t$. Thorne has posed the following question: In view of the fact that the gravitational interaction energy $E_{\text {int }}$ between the tidal field and the body is ambiguous by an amount $\sim \mathcal{E}_{j k} \mathcal{I}_{j k}$, is the tidal work also ambiguous by this amount, and therefore is the formula $d W / d t=-\frac{1}{2} \mathcal{E}_{j k} d \mathcal{I}_{j k} / d t$ only valid unambiguously when integrated over time scales long compared to that for $\mathcal{I}_{j k}$ to change substantially? This paper completes a demonstration that the answer is $n o ; d W / d t$ is not ambiguous in this way. More specifically, this paper shows that $d W / d t$ is unambiguously given by $-\frac{1}{2} \mathcal{E}_{j k} d \mathcal{I}_{j k} / d t$ independently of one's choice of how to localize gravitational energy in general relativity. This is proved by explicitly computing $d W / d t$ using various gravitational stress-energy pseudotensors (Einstein, Landau-Lifshitz, Møller) as well as Bergmann's conserved quantities which generalize many of the pseudotensors to include an arbitrary function of position. A discussion is also given of the problem of formulating conservation laws in general relativity and the role played by the various pseudotensors.
\end{abstract}

DOI: 10.1103/PhysRevD.63.064013

PACS number(s): 04.20.Cv, 04.25.-g, 04.40.Dg, 04.70.-s

\section{INTRODUCTION AND SUMMARY}

For many gravitating two body systems in the universe, the primary means of energy transfer from one body to the other is through tidal work. This work is accomplished through the gravitational interaction between the tidal field of one body and the mass multipole moments of the other body. A simple example of this is the work that the moon does on the earth as it raises the ocean's tides. Tidal work is also dramatically evident in the moon Io, which gets heated as it travels in an elliptical orbit through Jupiter's tidal gravitational field. This heating is the cause of Io's dramatic volcanism. In these cases it is clear that the tidal work is a physical observable and should in no way depend on one's means of calculating it.

The term "tidal heating", is often used in place of "tidal work," but is something of a misnomer. The net gravitational-energy that is transfered between two bodies interacting tidally does not necessarily go into heat. It may go into the energy needed to deform the body (i.e., raise a tide on it) or it may go into the internal vibrational energy of the body. The net "tidal work" may also be negative, in which case the phrase "tidal cooling" might be more appropriate. Throughout this paper we will take the terms "tidal heating" and 'tidal work' to be equivalent and to mean the net work done by an external tidal field on an isolated body.

It seems evident that tidal work should be a "physical observable;" i.e., the net energy-transfer from one body to another should be a real, physical quantity and should not depend on the mathematics that one uses to calculate the work that is done.

In calculating the tidal work for situations in general rela-

\footnotetext{
*Present address: Department of Astronomy, Cornell University, Ithaca, NY 14853.
}

tivity, we consider the interaction between an isolated body and a complicated "external universe", (the precise definition of these terms will be discussed in Sec. III). In the most familiar cases, this external universe may simply refer to a gravitating body such as a star, a planet, or a black hole, that orbits around the isolated body. In such a system it has been shown by Thorne and Hartle [1] that the total mass-energy $M$ of the isolated body is ambiguous by an amount $\Delta M$ $\sim \mathcal{I}_{j k} \mathcal{E}_{j k}$, where $\mathcal{I}_{j k}$ is the mass quadrupole moment of the isolated body and $\mathcal{E}_{j k}$ is the tidal field of the external universe. This mass ambiguity has the same physical origin as the ambiguity in the localization of energy in a gravitational wave: It arises from the fact that there is no preferred way to localize gravitational energy. This is true in Newtonian gravitational theory as well as in general relativity [2].

This mass ambiguity shows up mathematically in the fact that the nonlinearity of Einstein's equations could be expected to produce a term $\sim \mathcal{E}_{j k} \mathcal{I}_{j k} / r$ in the time-time component $g_{00}$ of the spacetime metric outside the body (where $r$ is a radial coordinate); and one is free, mathematically, to move this term or some arbitrary part of it into the mass $M$ that appears in the standard equation $g_{00}=-1+2 M / r$ $+\cdots$.

We can also understand this mass-energy ambiguity physically in terms of the standard experiment by which the total mass-energy $M$ of a gravitating body is measured: the application of the general relativistic version of Kepler's law to a test particle in orbit around the body. If the body is spherical and isolated and the orbit is circular, the body's mass-energy is related to the orbit's period $T$ (as measured by distant clocks) and its radius $r$ (defined to be its circumference divided by $2 \pi)$ by $M=\left(r^{3} / G\right)(2 \pi / T)^{2}$. If the body is non-spherical, with various multipole moments including the quadrupole moment $\mathcal{I}_{j k}$, then the moments perturb the orbit; but if one makes the orbit as circular as those perturbations permit and measures the orbit's average radius $\bar{r}$, then the $M$ that appears in the monopole $1 / r$ part of the 
gravitational field is still given accurately, to first order in the moments, by the relativistic Kepler law $M$ $=\left(\bar{r}^{3} / G\right)(2 \pi / T)^{2}$. The perturbations, being non-monopolar, disappear when averaged over the orbit. Similarly, if the body is precisely spherical but is perturbed by a weak, external tidal field $\mathcal{E}_{j k}$, then accurate to first order in those perturbations we can still compute $M$ by this averaged-radius formula; again the perturbations average to zero over the orbit (provided that the timescale on which $\mathcal{E}_{j k}$ changes is long compared to the orbital period). However, if both a quadrupole moment $\mathcal{I}_{j k}$ and an external tidal field $\mathcal{E}_{j k}$ are present simultaneously, then the product $\mathcal{E}_{j k} \mathcal{I}_{j k}$ is monopolar in nature and has dimensions of mass; and correspondingly Kepler's law with an average radius will give $M$ $+\mathcal{O}\left(\mathcal{E}_{j k} \mathcal{I}_{j k}\right)$. Thus, one cannot measure $M$ directly by $\mathrm{Ke}-$ pler's law. We shall discuss this mass ambiguity further in Sec. III.

Zhang [3] has used the Landau-Lifshitz pseudotensor (one of an infinite number of ways to localize gravitational-field energy) to derive the expression

$$
\frac{d W}{d t}=-\frac{1}{2} \mathcal{E}_{j k} \frac{d \mathcal{I}_{j k}}{d t}
$$

for the rate at which a time-evolving tidal field $\mathcal{E}_{j k}(t)$ does work on a body with time-evolving quadrupole moment $\mathcal{I}_{j k}(t)$. In view of the body's mass ambiguity $\Delta M \sim \mathcal{E}_{j k} \mathcal{I}_{j k}$, Zhang (and also Thorne and Hartle [1]) asserted that the work done should be ambiguous by an amount $\sim \Delta M$, and thus Eq. (1) should instead be written as $d W / d t$ $=\left\langle-\frac{1}{2} \mathcal{E}_{j k} d \mathcal{I}_{j k} / d t\right\rangle$ and would be valid only when averaged over timescales long enough for $W$ to build up by an amount large compared to $\Delta M=\mathcal{E}_{j k} \mathcal{I}_{j k}$. This occurs, for example, in the long-term tidal heating of Io, during which $\mathcal{E}_{j k}$ and $d \mathcal{I}_{j k} / d t$ oscillate partially in phase with each other, producing a cumulative work that goes into heat.

More recently Thorne [4], while analyzing the effects of tidal forces on the stability of relativistic stars, claimed on physical grounds that Zhang [3] and Thorne and Hartle [1] were wrong: The ambiguity $\Delta M$ actually resides solely in the energy of gravitational interaction $E_{\text {int }}$ between the body and the external tidal field and not at all in the body's self energy $E_{\text {self }}$ (i.e., the total mass-energy contained within the volume of the body), and correspondingly not at all in the work done by the tidal field on the body, $W=$ (change in $E_{\text {self }}$ ); so the rate of work done is unambiguously and instantaneously given by $d W / d t=-\frac{1}{2} \mathcal{E}_{j k} d \mathcal{I}_{j k} / d t{ }^{1}$

An operational variant of Thorne's argument is this: Consider a body on which tidal work is being done by the interaction between its time-changing quadrupole moment $\mathcal{I}_{j k}(t)$ and some external time-changing tidal field $\mathcal{E}_{j k}(t)$. One can imagine, at any moment of time, turning off the tidal field while holding the body's size and shape unchanged (to first order in the tidal field). With the tidal field gone, we can

\footnotetext{
${ }^{1}$ Thorne [4] needed this result as a key underpinning of his proof that tidal coupling stabilizes a star against gravitational collapse.
}

imagine measuring the body's total mass-energy $M_{i}$ (e.g., by using the relativistic version of Kepler's laws). That measured mass-energy with tidal field (momentarily) gone can be regarded as the body's self-energy $E_{\text {self }}$ (including rest mass). This measured self-energy is unambiguous. Now, turn the tidal field back on and allow the system to evolve normally for some time $\Delta t$. Then, turn the tidal field off and make a second measurement of the body's total mass-energy $M_{f}$ in the same manner as before. The difference $M_{f}-M_{i}$ between these two measurements is the change in the body's self-energy $\Delta E_{\text {self }}$. This is the work $W$ done by the tidal field on the isolated body. We can then conclude that, since these measured changes in the body's self energy are unambiguous, $d W / d t=d E_{\text {self }} / d t$ and $W$ are also unambiguous.

It is possible to test Thorne's claim in a manner based on the following considerations: The self-energy, defined in the above manner, will not change when the tidal field $\mathcal{E}_{j k}(t)$ changes, but the shape and size of the body are held fixed and thence $\mathcal{I}_{j k}$ is held fixed. This is just a restatement of the fact that a force can do no work if there is no displacement. However, if $\mathcal{I}_{j k}$ changes, with $\mathcal{E}_{j k}$ held fixed, then $E_{\text {self }}$ can change. This means that the unambiguous tidal work must be of the form $d W / d t=d E_{\text {self }} / d t=($ some constant $) \times \mathcal{E}_{j k} d \mathcal{I}_{j k} /$ $d t$. The interaction energy, by contrast, should have the form of a product of the instantaneous tidal field and quadrupole moment, so its time rate of change should be a perfect time derivative $d E_{\text {int }} / d t=d / d t[$ ( some constant $\left.) \times \mathcal{E}_{j k} \mathcal{I}_{j k}\right]$. The body's total mass $M$ must be the sum of its self-energy and that portion of the interaction energy that resides inside and near the body (i.e., within the orbit of the test particle that one uses in applying Kepler's third law to compute the mass), therefore $d M / d t=d E_{\text {self }} / d t+d E_{\text {int }} / d t=d W / d t$ $+d E_{\text {int }} / d t$. If we express $d M / d t$ in the form

$$
\frac{d M}{d t}=(\text { const }) \times \mathcal{E}_{j k} \frac{d \mathcal{I}_{j k}}{d t}+(\text { const }) \times \frac{d\left[\mathcal{E}_{j k} \mathcal{I}_{j k}\right]}{d t},
$$

then the first term must be $d W / d t$ and the second $d E_{\mathrm{int}} / d t .^{2}$ If Thorne is correct in his claim that $d W / d t$ is unambiguous and that the total ambiguity of $d M / d t$ resides in $d E_{\mathrm{int}} / d t$, then any computation of $d M / d t$ using any (general relativistically acceptable) localization of gravitational energy must give $-1 / 2$ unambiguously for the coefficient of the first term, while different localizations should give different values for the coefficients of the second term.

Purdue [2] has carried out detailed calculations of $d M / d t$ in Newtonian theory using all possible localizations of the gravitational energy and has found that, indeed, the first term in Eq. (2) always has the coefficient $-1 / 2$ while the second depends on the localization. Purdue has also verified that in general relativity, if one uses the energy localization embodied in the Landau-Lifshitz pseudotensor, but performs gauge transformations (infinitesimal coordinate transformations) on the spacetime metric, the first coefficient (that associated

\footnotetext{
${ }^{2}$ After this paper was submitted, it came to the attention of the author that Mashhoon [5] has derived an equation of this form.
} 
with $d W / d t$ ) remains always $-1 / 2$, while the second (that associated with $d E_{\text {int }} / d t$ ) changes with the changing gauge.

In this paper we shall complete this test of Thorne's claim: We shall verify that, when one changes the general relativistic energy localization by changing one's choice of pseudotensor, the first coefficient in Eq. (2) remains always $-1 / 2$ while the second changes and thus (partially) embodies the ambiguities present in localizing gravitational-field energy.

As a foundation for demonstrating this we first discuss in Sec. II of this paper the problem of formulating covariant conservation laws in general relativity. This is an underlying issue throughout this paper as the lack of an acceptable energy-momentum tensor for the gravitational field could possibly be a source of ambiguity in the calculation of the tidal work. We also discuss some of the various pseudotensors and conserved quantities that are used to describe gravitational-energy localization.

In Sec. III we discuss the assumptions that go into our calculation of the tidal work. A key issue is that our calculations are performed in the local asymptotic rest frame (LARF) of the body on which the work is being done. This means we are able to formulate only approximate conservation laws for our system. These laws are formulated in a buffer zone where the gravity of the isolated body is weak and the tidal field of the external universe is uniform. The spacetime metric of this buffer zone is described in Sec. III A.

We then calculate the tidal work using the Einstein pseudotensor (Sec. III B) and review the calculation given by Purdue [2] using the Landau-Lifshitz pseudotensor (Sec. III C). In Sec. III D we perform the calculation using the pseudotensor of Mbller [6], which is significantly different from the two previously mentioned pseudotensors, and in Sec. III E we examine the calculation using the conserved quantities found by Bergmann [7]. Bergmann's conserved quantities generalize many of the pseudotensors, including those of Landau and Lifshitz, and Einstein. Each of these calculations gives the same, standard result $d W / d t=$ $-\frac{1}{2} \mathcal{E}_{j k} d \mathcal{I}_{j k} / d t$ for the tidal work, in agreement with Thorne's assertion.

Throughout this paper we adhere to the conventions of Misner, Thorne, and Wheeler (MTW) [8]. Space-time indices are represented by Greek letters and spatial indices by Latin letters. We use units where $G=c=1$. The constant on the right-hand side of the Einstein field equations is $+8 \pi$ and the Minkowski flat-space metric $\eta_{\alpha \beta}$ has signature ( - , $+,+,+)$.

\section{CONSERVATION LAWS AND PSEUDOTENSORS}

The formulation of covariant conservation laws has been a problematic issue since general relativity's formulation in 1916. The issue has been addressed by a large number of authors and some continue to work on this problem.

If one considers a system without gravitational fields, as in special relativity, then the differential conservation laws for all matter and energy fields present are given by the familiar formula

$$
T^{\mu \nu}{ }_{, \nu}=0,
$$

where $T^{\mu \nu}$ is the symmetric energy-momentum tensor of matter that appears as a source term on the right-hand side of the Einstein field equations

$$
G^{\mu \nu}=8 \pi T^{\mu \nu} .
$$

By matter we mean all fields with the exception of the gravitational field.

In general relativity Eq. (3) is not an acceptable conservation law as it is not a tensor equation valid in all reference frames. Instead we must use the covariant derivative in place of the partial derivative and our equation becomes

$$
T_{; \nu}^{\mu \nu}=T_{, \nu}^{\mu \nu}+T^{\sigma \nu} \Gamma_{\sigma \nu}^{\mu}+T^{\mu \sigma} \Gamma_{\sigma \nu}^{\nu}=0,
$$

where $\Gamma_{\sigma \nu}^{\mu}$ are the connection coefficients. From Eq. (5) we can see that the mass-energy in matter fields is no longer conserved as energy can now be transfered between the matter and the gravitational field. The quantity that is actually conserved in the sense of Eq. (3) is some effective energymomentum tensor $T_{\text {eff }}^{\mu \nu}$ of matter plus gravitational fields which is given (in one variant) by Eq. (20.18) of MTW [8] as

$$
T_{\mathrm{eff}}^{\mu \nu}=T^{\mu \nu}+t^{\mu \nu},
$$

where $t^{\mu \nu}$ is an energy-momentum pseudotensor for the gravitational field. In other variants, some of which are encountered below, $T_{\mathrm{eff}}^{\mu \nu}=(-g)^{n / 2}\left(T^{\mu \nu}+t^{\mu \nu}\right)$, where $g$ $=\operatorname{det}\left\|g_{\alpha \beta}\right\|$ and $n$ is a positive integer. ${ }^{3}$ For each of these $T_{\text {eff }}^{\mu \nu}$, the equation $T^{\mu \nu}{ }_{; \nu}=0$ can be rewritten as

$$
T_{\mathrm{eff}, \nu}^{\mu \nu}=0,
$$

and $T_{\text {eff }}^{\mu \nu}$ can be written as the divergence of some "superpotential" $H^{\mu[\nu \sigma]}$ that is antisymmetric in its last two indices [9]:

$$
T_{\mathrm{eff}}^{\mu \nu}=H^{\mu[\nu \sigma]}, \sigma \cdot
$$

Square brackets indicate antisymmetry of the tensor when the enclosed indices are swapped. Notice that Eq. (7) follows from Eq. (8) by differentiation and symmetry.

As mentioned above, $t^{\mu \nu}$ is not a true tensor, but rather is a pseudotensor that describes the localization of gravitational energy-momentum. That $t^{\mu \nu}$ is not a tensor is a fact intimately linked with Einstein's equivalence principle. Since we are always free to choose our coordinates in spacetime to correspond to a freely falling frame where the acceleration vanishes at a point, we can equivalently choose a frame where the gravitational field vanishes at that point. In such a frame all the components of $t^{\mu \nu}$ will likewise vanish at that point (provided one is using Minkowski coordinates). However, in any other reference frame, there is no reason why all

\footnotetext{
${ }^{3} \mathrm{We}$ will sometimes refer to scalars, vectors and tensors with factors of $(-g)^{n / 2}$ in front as scalar, vector and tensor densities of weight $n$.
} 
the components of $t^{\mu \nu}$ should vanish. Since any tensor that vanishes in one reference frame must vanish in all reference frames, we can conclude that $t^{\mu \nu}$ is not a tensor but a pseudotensor, and quantities calculated from it will depend on the choice of one's coordinate system. To make matters worse, $t^{\mu \nu}$ is defined only up to a vanishing divergence, so there are an infinity of expressions for $t^{\mu \nu}$ corresponding to an infinite number of ways in which one can localize the gravitational energy-momentum density.

Despite their rather unpleasant nature in a theory so firmly rooted in the principle of general covariance, pseudotensors have proved to be rather valuable calculational tools, especially in gravitational-wave research (see for example [10]). The reason is that, despite their noncovariance, the $T_{\text {eff }}^{\mu \nu}$ can be used to compute covariant conserved quantities. For example, one can compute the total 4-momentum of a system that resides alone in asymptotically flat spacetime by the volume integral

$$
P^{\mu}=\int T_{\text {eff }}^{\mu 0} d^{3} x,
$$

where $d^{3} x=d x^{1} d x^{2} d x^{3}$ is a 3 -volume element of constant time. Even though the integrand depends highly on one's choice of coordinates, $P^{\mu}$ is a true vector that resides in the asymptotically flat region.

Using Gauss' law and the antisymmetry properties of the superpotential, it is also possible to express the 4-momentum as a surface integral:

$$
P^{\mu}=\int H_{, \sigma}^{\mu[0 \sigma]} d^{3} x=\oint H^{\mu[0 j]} n_{j} d^{2} S,
$$

where $n_{j}$ is the unit normal vector to the surface $S$. It is important to note that these integrals must be evaluated using an asymptotically Lorentz coordinate system. ${ }^{4}$

\section{A. The Einstein pseudotensor}

The first pseudotensor was formulated by Einstein in 1916. The Einstein pseudotensor is often referred to as the "canonical" pseudotensor because it is derived using the general formula for the energy-momentum tensor of a classical field with Lagrangian density $\mathcal{L}$ and field variables $\eta_{A}$, which may be tensors of any rank. In flat spacetime this general formula is given by (see, e.g., Goldstein [12]),

$$
T_{\mu \nu}=\frac{\partial \mathcal{L}}{\partial \eta_{A, \nu}} \eta_{A, \mu}-\mathcal{L} \delta_{\mu \nu}
$$

and the Euler-Lagrange equations guarantee that $T^{\mu \nu}{ }_{\nu}=0$. In general relativity, the field variables are the components of the metric tensor $g_{\mu \nu}$, and the Lagrangian density is given by

\footnotetext{
${ }^{4}$ However, it should be noted that Nahmad-Achar and Schutz [11] have devised a prescription for calculating pseudotensor-based conserved quantities for isolated systems in general relativity using coordinate systems with arbitrary asymptotic behavior.
}

$$
\mathcal{L}=\frac{1}{16 \pi} \sqrt{-g} g^{\alpha \beta}\left(\Gamma_{\alpha \beta}^{\gamma} \Gamma_{\gamma \sigma}^{\sigma}-\Gamma_{\alpha \gamma}^{\sigma} \Gamma_{\beta \sigma}^{\gamma}\right)
$$

Equation (11) then becomes

$$
{\sqrt{-g_{\mathrm{E}}}}_{t_{\mu}}{ }^{\nu}=\left(\frac{\partial \mathcal{L}}{\partial g_{\alpha \beta, \nu}} g_{\alpha \beta, \mu}-\delta_{\mu}{ }^{\nu} \mathcal{L}\right),
$$

and again the Euler-Lagrange equations guarantee that $\left(\sqrt{-g} t_{\mathrm{E}}{ }_{\mu}{ }^{\nu}\right)_{, \nu}=0$ in vacuum and ${ }_{\mathrm{E}} \mathfrak{T}_{\mu}{ }^{\nu}{ }_{, \nu}=0$ where

$$
\mathrm{E}_{\mu}{ }^{\nu}=\sqrt{-g}\left(T_{\mu}{ }^{\nu}+{ }_{\mathrm{E}} t_{\mu}{ }^{\nu}\right)
$$

when matter is present. The tensor density $\mathrm{E}^{\mathfrak{T}}{ }_{\mu}{ }^{\nu}$ is often referred to as a "total energy-momentum complex;" it is the Einstein variant of the $T_{\text {eff }}^{\mu \nu}$ discussed above.

From Eq. (13) we arrive at an explicit expression for the Einstein pseudotensor [13]:

$$
\sqrt{-g}_{\mathrm{E}} t_{\mu}{ }^{\nu}=\frac{1}{16 \pi}\left(\left(\Gamma^{\nu}{ }_{\alpha \beta}-\delta_{\beta}{ }^{\nu} \Gamma_{\alpha \sigma}^{\sigma}{ }_{\alpha \sigma}\right)\left(g^{\alpha \beta} \sqrt{-g}\right)_{, \mu}-\delta_{\mu}{ }^{\nu} \mathcal{L}\right) .
$$

Note that raising or lowering an index for this pseudotensor does not produce a symmetric quantity, so we are unable to form a conserved angular-momentum complex from it.

It was shown by von Freud [14] that the Einstein complex can be written as the divergence of an antisymmetric "superpotential" ${ }_{\mathrm{F}} U_{\alpha}^{[\beta \gamma]}$ :

$$
\mathrm{E}^{\mathfrak{T}_{\mu}{ }^{\nu}={ }_{\mathrm{F}} U_{\mu}^{[\nu \sigma]}, \sigma}
$$

where

$$
{ }_{\mathrm{F}} U_{\alpha}^{[\beta \gamma]}=-\frac{1}{16 \pi} \frac{g \alpha \sigma}{\sqrt{-g}}\left\{-g\left(g^{\beta \sigma} g^{\gamma \lambda}-g^{\gamma \sigma} g^{\beta \lambda}\right)\right\}_{, \lambda} .
$$

We can now form expressions for the covariant components of the 4-momentum of an isolated system by means of Eqs. (9) and (10):

$$
P_{\mu}=\int \mathrm{E}_{\mu}{ }^{0} d^{3} x=\oint_{\mathrm{F}} U_{\mu}^{[0 j]} n_{j} d^{2} S,
$$

where the first integral is over the system's entire volume, and the second is over a closed surface near spatial infinity (in the asymptotically flat region of spacetime). Because of the peculiarities of the pseudotensor, the above integral can only be interpreted as the covariant components of an energy-momentum 4-vector if one is using coordinates $x^{\mu}$ $=(t, x, y, z)$ in which the metric $g_{\alpha \beta}$ asymptotically approaches the Minkowski flat metric $\eta_{\alpha \beta}$.

To illustrate the coordinate dependent nature of the pseudotensors, we provide two well known examples (mentioned by Mфller [6] and Anderson [15]). If one were to calculate the integral $\int_{\mathrm{E}} t_{0}{ }^{0} d^{3} x$ for the Lorentz metric $g_{\alpha \beta}$ $=\eta_{\alpha \beta}$, its value would be zero, the expected energy for a region with no gravitational field. However, if we merely change to spherical coordinates, the value of this integral is 
infinite even though spacetime is flat. Similarly, evaluation of the integral for the Schwarzschild metric only yields the mass $M$ if one uses coordinates such that $g_{\alpha \beta}$ maps to $\eta_{\alpha \beta}$ asymptotically as $r \rightarrow \infty$.

Despite the restrictions on the use of this pseudotensor, it has still led to the reliable prediction by Einstein that gravitational waves exist and carry a definite energy.

\section{B. The Landau-Lifshitz pseudotensor}

Landau and Lifshitz (LL) [9] were able to formulate a symmetric pseudotensor, thus allowing the construction of a conserved total angular-momentum complex. Their conserved total energy-momentum complex (their variant of $T_{\text {eff }}^{\mu \nu}$ ) is given by

$$
\mathfrak{T}_{\mathrm{LL}}^{\mu \nu}=h^{\mu[\nu \sigma]}, \sigma=(-g)\left(T^{\mu \nu}+t_{\mathrm{LL}}^{\mu \nu}\right),
$$

(where $h^{\mu[\nu \sigma]}$ is defined below) and satisfies the usual property

$$
\mathfrak{T}_{\mathrm{LL}, \nu}^{\mu \nu}=0
$$

The explicit form of $t_{\mathrm{LL}}^{\mu \nu}$ is long and complicated. It is given by

$$
\begin{aligned}
(-g) t_{\mathrm{LL}}^{\mu \nu} & =[\text { Eq. }(20.23) \text { of MTW }] \\
& =[\text { Eq. }(96.9) \text { of LL }] .
\end{aligned}
$$

The LL superpotential $h^{\mu[\nu \sigma]}$ is related to that given by von Freud by [15]

$$
h^{\mu[\nu \sigma]}=\sqrt{-g} g_{F}^{\mu \rho} U_{\rho}^{[\nu \sigma]} .
$$

The LL pseudotensor is related to the Einstein pseudotensor by the following formula [15]:

$$
(-g) t_{\mathrm{LL}}^{\mu \nu}=(-g) g^{\mu \rho} \mathrm{E}_{\rho} t^{\nu}+\left(\sqrt{-g} g^{\mu \rho}\right)_{, \sigma \mathrm{F}} U_{\rho}^{[\nu \sigma]} \text {. }
$$

As in the case with the Einstein pseudotensor, integrals of the Landau-Lifshitz pseudotensor also produce strange results in curvilinear coordinate systems. Asymptotically Lorentz coordinates must again be used if one wants sensible results.

\section{The Mbller pseudotensor}

The Møller pseudotensor [6] is significantly different from the two complexes mentioned above. In deriving his pseudotensor Møller sought to eliminate the problem that the integral given in Eq. (18) yields strange results if one converts to curvilinear coordinate systems.

To define his pseudotensor, Møller makes use of the fact that one can always add a quantity $S_{\mu}{ }^{\nu}$ to the Einstein complex and still retain energy-momentum conservation, provided that $S_{\mu}{ }^{\nu}{ }_{\nu}=0$. The new total pseudotensor complex (matter plus gravitational fields) will thus have the form

$$
\mathrm{M}^{\mathfrak{T}_{\mu}}{ }^{\nu}={ }_{\mathrm{E}} \mathfrak{T}_{\mu}{ }^{\nu}+S_{\mu}{ }^{\nu}
$$

Møller additionally restricts the form of ${ }_{\mathrm{M}} \mathfrak{T}_{\mu}^{\nu}$ by requiring the following conditions (see also Komar [16]):

(1) It must be identically conserved: $\mathrm{M}^{\mathfrak{T}}{ }_{\mu}{ }^{\nu}, \nu=0$.

(2) The integral over some 3-volume of constant time of $\mathrm{M}^{\mathfrak{T}_{\mu}}{ }^{\nu}$ must produce the same results (in an asymptotically Lorentz coordinate system) as Eq. (18):

$$
\int \mathrm{M}_{\mu}{ }^{0} d^{3} x=\int \mathrm{E}_{\mu}{ }^{0} d^{3} x
$$

(3) $\mathrm{M}^{\mathfrak{T}_{0}}{ }^{0}$ and $\mathrm{M}^{\mathfrak{T}_{0}}{ }^{\nu}$ behave like scalar and vector densities under arbitrary changes of the spatial coordinates, $x_{\text {new }}^{j}$ $=F^{j}\left(x_{\text {old }}^{1}, x_{\text {old }}^{2}, x_{\text {old }}^{3}\right), x_{\text {new }}^{0}=x_{\text {old }}^{0}$. This allows one to change the coordinate system from say, Minkowski to spherical, but not to change the way one slices spacetime into space plus time.

(4) Under linear transformations, $\mathrm{M}^{\mathfrak{T}_{\mu}}{ }^{\nu}$ behaves like a mixed second-rank tensor.

With the further restriction that it not contain higher than second order derivatives of the metric, Møller explicitly exhibits a unique energy-momentum complex with these properties, and he shows that it can be written as the divergence of the following antisymmetric superpotential $\chi_{\mu}{ }^{[\nu \sigma]}$ :

$$
\mathrm{M}^{\mathfrak{T}_{\mu}}{ }^{\nu}=\frac{\partial \chi_{\mu}^{[\nu \sigma]}}{\partial x^{\sigma}}=-\frac{1}{8 \pi} \frac{\partial}{\partial x^{\sigma}}\left[\sqrt{-g}\left(g_{\mu \alpha, \beta}-g_{\mu \beta, \alpha}\right) g^{\nu \beta} g^{\sigma \alpha}\right] \text {. }
$$

The Møller superpotential $\chi_{\mu}^{[\nu \sigma]}$ is related to the von Freud superpotential $[15,16]$ :

$$
\chi_{\mu}^{[\nu \sigma]}=2{ }_{\mathrm{F}} U_{\mu}^{[\nu \sigma]}-\delta_{\mu}{ }^{\nu}{ }_{\mathrm{F}} U_{\rho}^{[\rho \sigma]}+\delta_{\mu}{ }^{\sigma}{ }_{\mathrm{F}} U_{\rho}^{[\rho \nu]} .
$$

Like the Einstein pseudotensor, the Møller complex is not symmetric and thus cannot be used to form conservation laws for angular momentum. Moreover, unlike the complexes of Einstein and Landau-Lifshitz, the Møller complex is not entirely quadratic in the first derivatives of the metric but has a term that is linear in the second derivatives of the metric. As pointed out by Møller himself [6], this means that

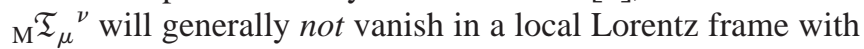
no matter present. This will become an issue in Sec. III, when we use the Møller complex to calculate the tidal work.

\section{The Bergmann conserved quantities}

Recognizing that conservation laws are related to the invariance properties of physical laws, and combining this with the fact that the equations of general relativity are invariant under arbitrary coordinate transformations, Bergmann [7] proposed that to each infinitesimal coordinate transformation there would correspond a conserved quantity. Making use of various identities, Bergmann [7] constructs a relationship between an arbitrary vector field $\xi^{\sigma}$ (which may be thought of as producing infinitesimal coordinate transformations on the metric) and the generators $C^{\rho}$ of these transformations:

$$
\sqrt{-g} G^{\mu \nu} \delta g_{\mu \nu}+C_{, \rho}^{\rho} \equiv 0,
$$

where 


$$
\delta g_{\mu \nu}=-\left(\xi_{\mu ; \nu}+\xi_{\nu ; \mu}\right) .
$$

Bergmann chooses $C^{\rho}=2 \sqrt{-g} G^{\rho \sigma} \xi_{\sigma}$ as a solution to Eq. (29). However, one may always add an arbitrary curl field $V^{[\rho \sigma]}{ }_{\sigma}$ to $C^{\rho}$ and still satisfy Eq. (29). Bergmann chooses this curl such that the resulting expression contains no higher than first derivatives of the metric. His final expression satisfying Eq. (29) is

$$
\bar{C}^{\mu}=2 \xi^{\sigma} \sqrt{-g} G_{\sigma}^{\mu}+\left(\xi_{F}^{\sigma} U_{\sigma}^{[\mu \nu]}\right)_{, \nu} .
$$

Equation (31) represents a weakly conserved quantity, meaning that it satisfies $\bar{C}^{\mu}{ }_{, \mu}=0$ whenever the vacuum field equations are satisfied $\left(G^{\mu \nu}=0\right)$. The corresponding strong conservation law is $D_{, \mu}^{\mu}=0$, where

$$
D^{\mu} \equiv\left(\xi_{\mathrm{F}}^{\sigma} U_{\sigma}^{[\mu \nu]}\right)_{, \nu} \equiv \bar{C}^{\mu}-2 \sqrt{-g} G_{\sigma}^{\mu} \xi^{\sigma},
$$

as can be easily shown if we make use of the antisymmetry of ${ }_{\mathrm{F}} U_{\sigma}^{[\mu \nu]}$ and the commutativity of partial derivatives.

From this strongly conserved quantity Bergmann constructs the 4-momentum in the same manner as we did from Eq. (9):

$$
P^{\mu}=\int D^{\mu} d^{3} x
$$

Using Gauss' law and the antisymmetry of ${ }_{\mathrm{F}} U_{\sigma}^{[\mu \nu]}$, we can write the $\mu=0$ component as a surface integral,

$$
P^{0}=\oint \xi_{\mathrm{F}}^{\sigma} U_{\sigma}^{[0 j]} n_{j} d^{2} S .
$$

According to Bergmann, from the weakly conserved quantities $\bar{C}^{\mu}$, expressions equivalent to several of the pseudotensors can be derived by making specific choices for $\xi^{\sigma}$. For example choosing $\xi^{\sigma}=k^{\sigma}$, where $k^{\sigma}$ is a constant vector, yields the canonical Einstein expression contracted with $k^{\sigma}$, while setting $\xi^{\sigma}=\sqrt{-g} g^{\sigma \alpha} k_{\alpha}$ yields the LandauLifshitz expression contracted with $k^{\sigma}$. We have been unable to find a similar choice that yields the Møller pseudotensor. We believe that this is due to Bergmann's choice of the curl field $V^{[\rho \sigma]}{ }_{\sigma}$ containing no second derivatives of the metric. Recall that unlike most other pseudotensors, the Møller complex contains second derivatives of the metric.

\section{E. Other formulations of the conservation laws}

Aside from the method of using pseudotensors to formulate conservation laws in general relativity, there exist several other approaches as well. One of these is the method of quasilocal energy, a covariant definition of energy that arises from a Hamiltonian formulation of general relativity. For a discussion of the equivalence of the quasilocal and pseudotensor approaches to gravitational energy-momentum, see Chang, Nester, and Chen [17]. In parallel with this present research, Booth and Creighton [18] have calculated the tidal work using the Brown and York [19] quasilocal energy approach, and have arrived at the same result as we deduce below using various pseudotensors: $d W / d t=-\frac{1}{2} \mathcal{E}_{j k} d \mathcal{I}_{j k} / d t$.
There have also been efforts to find an energy-momentum tensor for the gravitational field. Shortly after relativity was formulated, Lorentz and Levi-Civita proposed that the Einstein tensor $G^{\mu \nu}$ be used as a gravitational energymomentum tensor. This however, did not prove fruitful. ${ }^{5}$ Most recently, Babak and Grishchuk [21] have shown that when one formulates general relativity as a non-linear field theory in flat spacetime, then there exists an energymomentum tensor for the gravitational field that has all the nice properties one might wish. This energy-momentum tensor is the Landau-Lifshitz pseudotensor with partial derivatives replaced by covariant derivatives with respect to the flat-background metric.

\section{CALCULATION OF THE TIDAL WORK}

In calculating the tidal work, we consider a system consisting of an isolated body that interacts with a complicated external universe in the slow-motion approximation. The body is isolated in the sense that the radius of curvature $\mathcal{R}$ of the external universe and the length scale $\mathcal{L}$ on which this curvature changes must both be large when compared with the size $R$ of the isolated body: $R / \mathcal{R} \ll 1$ and $R / \mathcal{L} \ll 1$. This means that the external universe is not subjecting the isolated body to very strong gravitational fields (as would happen e.g., in a neutron star and black hole close to merger) and that the tidal field of the external universe is nearly uniform in the region near the isolated body. By slow-motion, we mean that the time scale $\tau$ for changes in the mass and current moments of the body and the tidal field of the external universe are small compared to the size of the body: $R / \tau$ $\ll 1$. If this were not the case, we would have to worry about changes in the mass-energy $M$ due to gravitational radiation and other higher-order effects. For detailed discussions of the constraints $R / \mathcal{R} \ll 1, R / \mathcal{L} \ll 1$, and $R / \tau \ll 1$, and of various approximations based on them (which we shall use below), see Thorne and Hartle [1] and the recent paper by Purdue [2], whose analysis we are continuing.

Some examples of isolated, slow-motion bodies discussed by Purdue [2] include (i) a compact object such as a neutron star or black hole in a binary inspiraling system that is not too close to merger; and (ii) Jupiter's moon Io, which gets tidally heated as it travels through Jupiter's tidal field in an elliptical orbit.

Our calculation of the tidal work involves computing $d M / d t$, the rate of change of the mass of an isolated body, and then expressing $d M / d t$ in the form of Eq. (2) and reading off the two coefficients. We use the multipole moment formalism discussed in Thorne [22], and Thorne and Hartle [1], and treat gravity as a non-linear field theory in flat spacetime. The computation of $d M / d t$ is carried out as a 2-dimensional surface integral of a pseudotensor in the "buffer zone" or local asymptotic rest frame (LARF) of the

\footnotetext{
${ }^{5}$ For an excellent discussion of the exchange that occurred between Einstein, Levi-Civita, Lorentz, and others concerning conservation laws and the prediction of gravitational waves, see the article by Cattani and De Maria [20].
} 
isolated body [see Eq. (2.3a) of Thorne and Hartle [1]]:

$$
\frac{d M}{d t}=-\oint t^{0 j} d^{2} S_{j}
$$

where $d^{2} S_{j}=n_{j} r^{2} d \Omega$ is the surface element of a 2-sphere $\partial \mathcal{V}$ in the buffer zone that encloses a volume $\mathcal{V}$ and has unit normal $n_{j}$ and solid angle $d \Omega$. This buffer zone is a region that surrounds the isolated body but is far enough away that gravity in it can be considered weak. At the same time, it is close enough to the body that the tidal field of the external universe appears homogeneous. The buffer zone can be described more precisely [2] as the region where $r / \mathcal{L} \ll 1$, $r / \mathcal{R} \ll 1$, and $M / r \ll 1, r$ being the radial distance from the isolated body. The rate of change of mass-energy through the surface $\partial \mathcal{V}$ is $d M / d t$, and $M$ is the total mass-energy inside $\mathcal{V}$. Note that our analysis is thus valid even for a strongly gravitating body such as a black hole, provided there exists a buffer region around it where gravity is weak, the external curvature is nearly uniform, and the spacetime curvature is not changing too rapidly.

For the purposes of our discussion and to the order that our calculations are valid, there are only three relevant parameters that characterize the spacetime:

(1) The total mass-energy $M$ of the isolated body.

(2) The quadrupole moment $\mathcal{I}_{j k}$ of the isolated body, which, in the limit of weak gravity, is given by

$$
\mathcal{I}_{j k}=\int\left(\rho x_{j} x_{k}-\frac{1}{3} \delta_{j k} r^{2}\right) d^{3} x .
$$

(3) The tidal field of the external universe is given by $\mathcal{E}_{j k}=R_{j 0 k 0}$, where $R_{\alpha \beta \gamma \delta}$ is the Riemann tensor of the external universe.

Note that both $\mathcal{I}_{j k}$ and $\mathcal{E}_{j k}$ are symmetric and trace free tensors that reside in the buffer zone, and that we are using coordinates that are as Lorentz as possible (with respect to the physical metric) throughout the buffer zone; i.e., $g_{\alpha \beta}$ $=\eta_{\alpha \beta}+O(M / r)+O\left(\mathcal{I} / r^{3}\right)+O\left(\mathcal{E} r^{2}\right)$. These $\mathcal{I}_{j k}$ and $\mathcal{E}_{j k}$ are spatially constant in the buffer region but they may depend on time.

The body has additional multipole moments: the current quadrupole moment $\mathcal{S}_{j k}$, the mass octupole moment $\mathcal{I}_{j k l}$, etc; and the external universe has additional tidal fields of "magnetic-type" $\left(\mathcal{B}_{j k}, \ldots\right)$ and "electric-type" $\left(\mathcal{E}_{j k l}, \ldots\right)$, see e.g., Thorne and Hartle [1]. These moments and tidal fields can couple to each other to produce tidal work: $d W / d t \sim \mathcal{B}_{j k} d \mathcal{S}_{j k} / d t \& \mathcal{E}_{j k l} d \mathcal{I}_{j k l} / d t \& \cdots{ }^{6}$ In some situations these contributions to $d W / d t$ might be larger than the one, $d W / d t \sim \mathcal{E}_{j k} d \mathcal{I}_{j k} / d t$, that we are studying, but typically the mass quadrupole will dominate. In this paper we restrict ourselves to the mass quadrupole term, whether or not it dominates the tidal work, because we are seeking to discuss an issue of principle first raised by Thorne and Hartle [1]: the non-ambiguity of the $\mathcal{E}_{j k} d \mathcal{I}_{j k} / d t$ tidal work. Presum-

\footnotetext{
${ }^{6}$ Here, the symbol " $\&$ " means "plus terms of the form..."; see Thorne and Hartle [1].
}

ably our results can be generalized to higher order moments, but we shall not attempt to do so here. Correspondingly, in our analysis we shall consider only $M, \mathcal{I}_{j k}$, and $\mathcal{E}_{j k}$.

Keep in mind that when we identify a mass $M$ as in Eq. (35), we are only doing so in an approximate sense. This is because the mass, momentum, and angular momentum only have precisely defined values in an asymptotically flat spacetime. As our spacetime is only locally asymptotically flat, the conservation laws only give approximate values of the mass, momentum, and angular momentum in the buffer zone where spacetime is approximately asymptotically flat.

In particular, as we discussed in Sec. I, there is an ambiguity in the mass $M$ of the isolated body $\Delta M \sim \mathcal{I}_{j k} \mathcal{E}_{j k}$. If $\mathcal{I}_{j k}$ is oscillating in time, then this ambiguity is of the same order as the amount of energy that is transfered between the isolated body and the external universe by tidal work during one period of oscillation. We can understand this ambiguity more clearly by examining the time-time part of the metric in the buffer zone [2]:

$$
g_{00}=-1+\frac{2 M}{r}+3 \mathcal{I}_{a b} \frac{x^{a} x^{b}}{r^{5}}+\cdots-\mathcal{E}_{a b} x^{a} x^{b}+\cdots,
$$

where $r$ is the distance from the center of the isolated body as measured in its local asymptotic rest frame. We have omitted terms involving higher order mass and tidal multipole moments (e.g., octupole moments $\mathcal{I}_{j k l}$ and $\mathcal{E}_{j k l}$ ) and also terms that are products of $M, \mathcal{I}_{j k}$, and $\mathcal{E}_{j k}$ which result from the nonlinearities of the Einstein field equations. One of these nonlinear terms has the form

$$
\delta g_{00} \sim \frac{\mathcal{I}_{j k} \mathcal{E}_{j k}}{r}
$$

which has the same form as $2 M / r$, that is, (monopole) $/ r$, and which has a coefficient that is gauge dependent. The similarity in structure between $M / r$ and $\left(\mathcal{I}_{j k} \mathcal{E}_{j k}\right) / r$ implies that it is possible to move portions of the gauge-dependent term given in Eq. (38) into or out of the $2 M / r$ term. One can interpret this as meaning that the mass $M$ that one reads off the metric is ambiguous by an amount on the order of

$$
\Delta M \sim \mathcal{I}_{j k} \mathcal{E}_{j k}
$$

Purdue [2] shows that this ambiguity is also present in Newtonian theory in the form of an ambiguous gravitational interaction-energy inside and near the body. More specifically: The total mass-energy $E_{\mathcal{V}}$ enclosed in the volume $\mathcal{V}$ can be expressed in Newtonian theory as

$$
E_{\mathcal{V}}=E_{\text {self }}+E_{\mathrm{e}}+E_{\text {int }},
$$

where $E_{\text {self }}$ is the isolated body's self-energy (which depends on the body's rest-mass and internal energy density distributions), $E_{\mathrm{e}}$ is the external field energy inside the volume $\mathcal{V}$ (which depends only on the external tidal field $\mathcal{E}_{j k}$ ), and $E_{\text {int }}$ is the interaction energy inside $\mathcal{V}$ and is given by 


$$
E_{\text {int }}=\left(\frac{2+\alpha}{10}\right) \mathcal{I}_{j k} \mathcal{E}_{j k}
$$

Here the coefficient $\alpha$ depends on one's choice of Newtonian energy-localization. For example (see Purdue [2]), the choice $\alpha=0$ localizes all of the gravitational energy in the field, so that the total gravitational energy is given by a volume integral of $(\nabla \Phi)^{2} /(8 \pi)$ (which should be familiar from electrostatics). Alternatively, the choice $\alpha=1 / 2$ localizes the gravitational energy entirely in the matter, so the totalgravitational energy is given by a volume integral of $\frac{1}{2} \rho \Phi$. Here $\rho$ is the mass density, so clearly it vanishes outside the material of a gravitating body.

From Eq. (40) we can see that a Newtonian calculation of $d E_{\mathcal{V}} / d t$ (which is analogous to our general-relativistic calculation of $d M / d t$ ) will include a term that is the total time derivative of the interaction term $E_{\text {int }}$. Our generalrelativistic calculation will also have an interaction term of this same form, where the coefficient in front depends on the energy-localization scheme. In the Newtonian case, Purdue shows that, despite the ambiguity of $d E_{\text {int }} / d t$, the rate of change of the body's self-energy $d E_{\text {self }} / d t$ is given unambiguously by the tidal work formula $d E_{\text {self }} / d t$ $=-\frac{1}{2} \mathcal{E}_{j k} d \mathcal{I}_{j k} / d t$. Our general relativistic analysis will produce this same conclusion.

\section{A. Metric in the buffer zone}

In our calculation we will consider general relativistic gravity not as a geometric phenomenon involving the curvature of spacetime, but rather as a non-linear field theory in flat spacetime. We treat the field variables (the metric components $g_{\alpha \beta}$ ) as a perturbative expansion in some dimensionless parameter $\varepsilon$ which is actually the gravitation constant, $G=1$ in our system of units. Thus, terms of $O(\varepsilon)$ are linear perturbations around flat spacetime; terms of $O\left(\varepsilon^{2}\right)$ are quadratic, etc. All raising and lowering of indices is done with the flat Minkowski metric $\eta_{\alpha \beta}$.

Our three parameters $M, \mathcal{I}_{j k}$, and $\mathcal{E}_{j k}$ can all be considered linear in $\varepsilon$. In our calculation of the tidal work, it is clear from the form of Eq. (2) that we may also need to consider terms in the metric that are quadratic in $\varepsilon$, as such terms may go as $\mathcal{I}_{j k} \mathcal{E}_{j k}$. We thus expand the metric up to quadratic order:

$$
g_{\alpha \beta}=\eta_{\alpha \beta}+\varepsilon h_{\alpha \beta}+\varepsilon^{2} k_{\alpha \beta},
$$

where $h_{\alpha \beta}$ contains terms that are linear in $M, \mathcal{I}_{j k}, \mathcal{E}_{j k}$, and their time derivatives, while $k_{\alpha \beta}$ contains terms that are products of any two of those three quantities and their first time derivatives (for example $M \mathcal{I}, \dot{\mathcal{I}}$, $\mathcal{I} \mathcal{E}, \dot{\mathcal{E}} \dot{\mathcal{E}}$, etc.). Any terms that are cubic or higher in the perturbation expansion $\varepsilon$ cannot contribute to the tidal work (which is itself of order $\varepsilon^{2}$ ) and can be discarded at any point in the calculation.

Since the mass quadrupole moment $\mathcal{I}_{j k}$ and the tidal field $\mathcal{E}_{j k}$ are spatially uniform in the buffer zone, the spatial gradients of these functions vanish. Furthermore, since $\partial_{t} \sim 1 / \tau$, the slow-motion approximation allows us to ignore all second and higher order time derivatives of $\mathcal{I}_{j k}$ and $\mathcal{E}_{j k}$.
We impose separate gauge conditions on the linear $h_{\alpha \beta}$ and quadratic $k_{\alpha \beta}$ parts of the metric. The linear part of the metric has been calculated by Zhang [3] in the de Donder gauge and is used by Purdue [2] in her analysis. The de Donder gauge in linear order ${ }^{7}$ is given by the condition that $\bar{h}^{\mu \nu}{ }_{, \nu}=0$, where $\bar{h}^{\mu \nu}$ is the trace reversed metric perturbation:

$$
\bar{h}^{\mu \nu} \equiv h^{\mu \nu}-\frac{1}{2} \eta^{\mu \nu} h
$$

In terms of the metric perturbation $h_{\alpha \beta}$ we can write this as the condition

$$
h_{, \nu}^{\mu \nu}=\frac{1}{2} h^{, \mu}
$$

where $h$ is the trace of $h_{\alpha \beta}$ :

$$
h=h_{\alpha}{ }^{\alpha}=\eta^{\alpha \beta} h_{\alpha \beta} .
$$

To the order we should need in the slow-motion approximation (neglecting second and higher order time derivatives) and ignoring higher order multipole moments $\left(\mathcal{I}_{j k l}, \mathcal{S}_{j k}, \mathcal{E}_{j k l}, \mathcal{B}_{j k}\right.$, etc. $)$, the linear part of the metric is given in Cartesian coordinates and de Donder gauge, accurate to $O(\varepsilon)$ in the buffer zone, by [3]

$$
\begin{aligned}
& h_{00} \equiv-2 \Phi=2 \frac{M}{r}+3 \frac{\mathcal{I}_{i j} x^{i} x^{j}}{r^{5}}-\mathcal{E}_{i j} x^{i} x^{j}, \\
& h_{0 j} \equiv A_{j}=-2 \frac{\dot{\mathcal{I}}_{j a} x^{a}}{r^{3}}-\frac{10}{21} \dot{\mathcal{E}}_{a b} x^{a} x^{b} x^{j}+\frac{4}{21} \dot{\mathcal{E}}_{j a} x^{a} r^{2}, \\
& h_{i j}=-2 \Phi \delta_{i j}=\delta_{i j}\left(2 \frac{M}{r}+3 \frac{\mathcal{I}_{i j} x^{i} x^{j}}{r^{5}}-\mathcal{E}_{i j} x^{i} x^{j}\right),
\end{aligned}
$$

where $\Phi$ is a scalar potential analogous to that of Newtonian gravity and $A_{j}$ is a vector potential that has no Newtonian analog.

This metric, by virtue of the de Donder gauge conditions and the approximations mentioned above, satisfies the following relations, which we shall use in our calculations below:

$$
\begin{aligned}
A_{j, 0} & =0, \\
A_{j, j} & =-4 \Phi_{, 0}, \\
h_{\mu \nu, \sigma}{ }^{\sigma} & =0, \\
h_{, \sigma}{ }^{\sigma} & =0 .
\end{aligned}
$$

\footnotetext{
${ }^{7}$ The general de Donder condition is given by $\mathfrak{g}^{\mu \nu}{ }_{, \nu}=0$, where $\mathfrak{g}^{\mu \nu}=\sqrt{-g} g^{\mu \nu}$.
} 
To compute the quadratic part of the metric $k_{\alpha \beta}$ one would have to solve the second order Einstein field equations. It turns out that for the pseudotensors we are considering, a suitable choice of gauge will make the direct calculation of $k_{\alpha \beta}$ unnecessary. This specific gauge will be discussed later.

Other formulas that will be useful in the calculations that follow are given below, accurate up to order $\varepsilon^{2}$ :

$$
\begin{aligned}
g^{\alpha \beta} & =\eta^{\alpha \beta}-\varepsilon h^{\alpha \beta}+\varepsilon^{2} h^{\alpha \gamma} h_{\gamma}{ }^{\beta}-\varepsilon^{2} k^{\alpha \beta}, \\
g^{\alpha \beta} g_{\gamma \beta} & =\delta_{\gamma}{ }^{\alpha}+O\left(\varepsilon^{3}\right), \\
-g=\operatorname{det}\left(g_{\alpha \beta}\right) & =1+\varepsilon h+\frac{1}{2} \varepsilon^{2}\left(h^{2}-h^{\alpha \beta} h_{\alpha \beta}\right)+\varepsilon^{2} k,
\end{aligned}
$$

where $k$ is the trace of $k_{\alpha \beta}$. Taylor expanding Eq. (55) about $\varepsilon$, we also have, accurate to order $\varepsilon^{2}$,

$$
\begin{aligned}
& \sqrt{-g}=1+\frac{1}{2} \varepsilon h+\frac{1}{2} \varepsilon^{2}\left(\frac{1}{4} h^{2}-\frac{1}{2} h^{\alpha \beta} h_{\alpha \beta}\right)+\frac{1}{2} \varepsilon^{2} k, \\
& \frac{1}{\sqrt{-g}}=1-\frac{1}{2} \varepsilon h+\frac{1}{2} \varepsilon^{2}\left(\frac{1}{4} h^{2}+\frac{1}{2} h^{\alpha \beta} h_{\alpha \beta}\right)-\frac{1}{2} \varepsilon^{2} k .
\end{aligned}
$$

We are now ready to compute the tidal work using various pseudotensors.

\section{B. Calculation of tidal work using the Einstein pseudotensor}

We wish to calculate the integral given by Eq. (35) for the rate of change of mass-energy of the isolated body using the Einstein pseudotensor $\sqrt{-g} t_{\mathrm{E}} t^{j}$ :

$$
\frac{d M}{d t}=-\oint \sqrt{-g} t_{\mathrm{E}_{0}}{ }^{j} n_{j} r^{2} d \Omega,
$$

where $n_{j}=x_{j} / r$ is the unit normal to a surface lying in the buffer zone at some radius $r$, and $d \Omega$ is the solid angle on that surface. If we look at the form of $\sqrt{-g}_{\mathrm{E}}{ }_{\mu}{ }^{\nu}$ given by Eq. (15), we can see that in order to obtain an expression that is accurate up to order $\varepsilon^{2}$ we only need to expand the metric to order $\varepsilon$. This means that we can ignore all terms appearing in Eqs. (42), (53)-(57) that go as $\varepsilon^{2}$. Equation (15) thus takes the form (in a general gauge):

$$
\begin{aligned}
\sqrt{-g} t_{\mathrm{E}}{ }_{\mu}{ }^{\nu}= & \frac{\varepsilon^{2}}{16 \pi}\left\{\frac{1}{2} h_{, \mu} h_{, \alpha}^{\nu \alpha}-\frac{1}{4} h_{, \mu} h^{, \nu}-h_{, \mu}^{\alpha \beta} h_{\alpha, \beta}^{\nu}\right. \\
& +\frac{1}{2} h_{, \mu}^{\alpha \beta} h_{\alpha \beta}{ }^{, \nu}-\frac{1}{4} h_{, \mu} h^{, \nu}+\frac{1}{2} h_{, \alpha} h_{, \mu}^{\alpha \nu} \\
& -\delta_{\mu}{ }^{\nu}\left(\frac{1}{2} h_{, \gamma} h_{, \alpha}^{\gamma \alpha}-\frac{1}{4} h_{, \gamma} h^{, \gamma}-\frac{1}{2} h^{\alpha \sigma, \gamma} h_{\alpha \gamma, \sigma}\right. \\
& \left.\left.+\frac{1}{4} h^{\alpha \sigma, \gamma} h_{\alpha \sigma, \gamma}\right)\right\} .
\end{aligned}
$$

Applying the de Donder gauge constraints (44), we see that the first and second and the seventh and eighth terms cancel each other.

From Eq. (58) we see that we only need to evaluate the $\mathrm{E}^{t_{0}}{ }^{j}$ terms. These terms evaluate ${ }^{8}$ to

$$
\sqrt{-g_{\mathrm{E}}} t_{0}{ }^{j}=\frac{1}{4 \pi} \Phi_{, 0} \Phi_{, j}
$$

Using Eq. (46) we see that

$$
\begin{aligned}
\Phi_{, 0} \Phi_{, j}= & \frac{15}{4} \mathcal{I}_{a b} \dot{\mathcal{E}}_{c d} \frac{x_{a} x_{b} x_{c} x_{d} x_{j}}{r^{7}}-\frac{3}{2} \mathcal{I}_{j a} \dot{\mathcal{E}}_{b c} \frac{x_{a} x_{b} x_{c}}{r^{5}} \\
& -\frac{3}{2} \dot{\mathcal{I}}_{a b} \mathcal{E}_{j c} \frac{x_{a} x_{b} x_{c}}{r^{5}} .
\end{aligned}
$$

Note that we have ignored terms that go like $M \dot{M}, \dot{M} \mathcal{I}, \dot{M} \mathcal{E}$, $\dot{\mathcal{I}} M, \dot{\mathcal{I}} \mathcal{I}, \dot{\mathcal{E}} M$, and $\dot{\mathcal{E}} \mathcal{E}$ as they do not contribute to the tidal work. That these terms do not contribute is apparent if one considers that the tidal work must arise due to a coupling between the mass multipole moments of the isolated body and the tidal field of the external universe. This, combined with dimensional considerations, implies that only terms of the form $\dot{\mathcal{E}} \mathcal{E}$ and $\mathcal{I} \dot{\mathcal{E}}$ can contribute to $d W / d t$. Also we do not worry about spatial indices being up or down since we are using Cartesian coordinates.

We must now evaluate the surface integral given in Eq. (58). Note that keeping our calculations accurate to order $\varepsilon^{2}$ justifies our setting the factor of $\sqrt{-g}$ on the left-hand side of Eq. (60) to unity. To perform the surface integrals over the terms in Eq. (61), we first note that since the multipole moments do not vary spatially in the buffer region, they can be pulled out of the integrals. The surface integrals that remain are all of the form

$$
\oint n_{a} n_{b} n_{c} \ldots n_{p} d \Omega,
$$

where $n_{a}=x_{a} / r$ is a component of a unit radial vector. Evaluating such integrals (see Sec. IIB of Thorne [22]) we finally arrive at

$$
\frac{d M}{d t}=-\frac{1}{2} \mathcal{E}_{j k} \frac{d \mathcal{I}_{j k}}{d t}+\frac{d}{d t}\left(\frac{3}{10} \mathcal{I}_{j k} \mathcal{E}_{j k}\right) .
$$

We can identify the second term in the equation above as the analog of the Newtonian interaction-energy given by Eq. (41) where the Einstein pseudotensor localization corresponds to the choice $\alpha=1$. That this term is in fact the derivative of an interaction-energy term is apparent if one considers that the interaction energy $E_{\text {int }}$ between an isolated body and the external universe must depend only on the instantaneous fields and can only be given by a product $\sim \mathcal{I}_{j k} \mathcal{E}_{j k}$. The rate of change of this interaction energy must

\footnotetext{
${ }^{8}$ Part of this calculation was performed using the tensor algebra package GRTENSORII [23].
} 
then be a perfect differential. Also, a term that goes like $\sim \mathcal{I}_{j k} \dot{\mathcal{E}}_{j k}$ could not contribute to the tidal heating since no work is done if the isolated body does not change (just as no work is done if a force is exerted but no displacement results); see Sec. I for further discussion. These facts indicate that the first term in Eq. (63) is the tidal work while the second term is the rate of change of the interaction energy between the external universe and the body; cf. Eq. (2).

\section{Calculation of tidal work using the Landau-Lifshitz pseudotensor}

The calculation of the tidal work using the LandauLifshitz pseudotensor is very similar to that for the Einstein pseudotensor shown above. This calculation was performed by Purdue [2] and we will only summarize her results here.

Since the Landau-Lifshitz pseudotensor, like the Einstein pseudotensor, is quadratic in the first derivatives of the metric, one only needs to expand $g_{\alpha \beta}$ to first order in $\varepsilon$. We thus only need to consider the linear part of the metric $h_{\alpha \beta}$ when evaluating the integral in Eq. (58) [where $\sqrt{-g} t_{\mathrm{E}} t^{\nu}$ is replaced by $\left.(-g) t_{\mathrm{LL}}^{\mu \nu}\right]$.

Evaluating this integral and keeping only terms that contribute to the tidal work (in the same manner as the previous section), Purdue arrives at

$$
\frac{d M}{d t}=-\frac{1}{2} \mathcal{E}_{j k} \frac{d \mathcal{I}_{j k}}{d t}+\frac{d}{d t}\left(-\frac{1}{10} \mathcal{I}_{j k} \mathcal{E}_{j k}\right) .
$$

We notice that changing the energy localization scheme from that of Einstein to that of Landau-Lifshitz has simply changed the coefficient of the second term, which we again identify as the derivative of the interaction energy. Note that the Landau-Lifshitz localization scheme is analogous to a choice of $\alpha=-3$ in the Newtonian interaction term. We also see that the tidal work term [the first term in Eq. (64)] has remained unaffected.

\section{Calculation of tidal work using the Mbller pseudotensor}

We shall now perform the calculation of $d M / d t$ once again, this time making use of the Møller pseudotensor. Since we are working in the vacuum buffer zone where $T^{\mu \nu}=0$, we can use Eq. (27) as the expression for the energy-momentum pseudotensor of the gravitational field. Note that Eq. (27) is actually the total conserved complex that we would use if non-gravitational fields were also present.

If we examine closely the form of Eq. (27) we will find that unlike the two previous pseudotensors discussed, the Mbller complex has a term that is linear in the second derivatives of the metric perturbations. This means that we will not only have terms like $h^{\alpha \beta}{ }_{, \mu} h_{\alpha \beta}{ }^{, \nu}$ but will also have terms like $h_{\mu}{ }^{\sigma}{ }_{, \sigma}{ }^{\nu}$ and $k_{\mu}{ }^{\sigma}{ }^{\nu}{ }^{\nu}$. This means that it is important that we expand the metric up to quadratic order in $\varepsilon$ as these terms that are linear in the second derivatives of the metric perturbation are actually quadratic in $\varepsilon$ and will thus contribute to the calculation of the tidal work.
Using the metric given by Eq. (42) in Eq. (27), we arrive at the form of the Mbller total energy-momentum pseudotensor, correct up to order $\varepsilon^{2}$, in a general gauge:

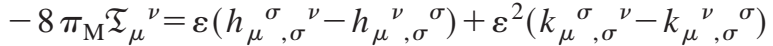

$$
\begin{aligned}
& +\frac{1}{2} \varepsilon^{2}\left(h_{, \sigma} h_{\mu}{ }^{\sigma, \nu}+h h_{\mu}{ }^{\sigma}{ }_{, \sigma}{ }^{\nu}-h_{, \sigma} h_{\mu}{ }^{\nu, \sigma}\right. \\
& \left.-h h_{\mu}{ }^{\nu}{ }_{, \sigma}{ }^{\sigma}\right)+\varepsilon^{2}\left(h^{\sigma \alpha}{ }_{, \sigma} h_{\mu}{ }^{\nu}{ }_{, \alpha}+h^{\sigma \alpha} h_{\mu}{ }^{\nu}, \alpha \sigma\right. \\
& -h^{\sigma \alpha}{ }_{, \sigma} h_{\mu \alpha}{ }^{\nu}-h^{\sigma \alpha} h_{\mu \alpha, \sigma}{ }^{\nu}+h^{\nu \beta}{ }_{, \sigma} h_{\mu \beta}{ }^{\sigma} \\
& \left.+h^{\nu \beta} h_{\mu \beta, \sigma}{ }^{\sigma}-h^{\nu \beta}{ }_{, \sigma} h_{\mu}{ }^{\sigma}{ }_{, \beta}-h^{\nu \beta} h_{\mu}{ }^{\sigma}{ }_{, \beta \sigma}\right) \text {. }
\end{aligned}
$$

If we work in de Donder gauge to linear order we can use Eqs. (46)-(48) for $h_{\alpha \beta}$. However, we still do not know the form of $k_{\alpha \beta}$.

Fortunately, we can make use of the vacuum Einstein field equations

$$
R_{\mu \nu}=\Gamma_{\mu \nu, \alpha}^{\alpha}-\Gamma_{\mu \alpha, \nu}^{\alpha}+\Gamma_{\beta \alpha}^{\alpha} \Gamma_{\mu \nu}^{\beta}-\Gamma_{\beta \nu}^{\alpha} \Gamma_{\mu \alpha}^{\beta}=0
$$

to solve for the derivatives of $k_{\alpha \beta}$ as they appear in Eq. (65). Specifically: If we substitute the metric (42) in Eq. (66) we can expand the Ricci tensor in powers of $\varepsilon$ :

$$
\varepsilon R_{\mu \nu}^{(1)}[h]+\varepsilon^{2} R_{\mu \nu}^{(2)}[h h]+\varepsilon^{2} R_{\mu \nu}^{(2)}[k]=0,
$$

where the superscript on the $R_{\mu \nu}^{(n)}$ means that the indicated piece of the Ricci tensor contains only terms of order $\varepsilon^{n}$. The terms in the brackets indicate that the part of the Ricci tensor in question contains terms that go like the indicated multiple of the metric piece $\left(h_{\alpha \beta}\right.$ or $\left.k_{\alpha \beta}\right)$ and its derivatives. Equations (70) and (71) make this clear. We now require that the vacuum field equations vanish in each order of $\varepsilon$ :

$$
\begin{aligned}
R_{\mu \nu}^{(1)}[h] & =0, \\
R_{\mu \nu}^{(2)}[h h]+R_{\mu \nu}^{(2)}[k] & =0 .
\end{aligned}
$$

The first of these equations yields the linearized vacuum field equations:

$$
h_{\mu, \nu \alpha}^{\alpha}+h_{\nu, \mu \alpha}^{\alpha}-h_{\mu \nu, \alpha}{ }^{\alpha}-h_{, \mu \nu}=0,
$$

while in the second (69) one can solve for $R_{\mu \nu}^{(2)}[k]$ to give

$$
\begin{aligned}
k^{\alpha}{ }_{\mu, \nu \alpha}+ & k^{\alpha}{ }_{\nu, \mu \alpha}-k_{\mu \nu, \alpha}{ }^{\alpha}-k_{, \mu \nu} \\
= & h^{\alpha \sigma}{ }_{, \alpha} h_{\sigma \mu, \nu}+h^{\alpha \sigma} h_{\sigma \mu, \nu \alpha}+h^{\alpha \sigma}{ }_{, \alpha} h_{\sigma \nu, \mu} \\
& +h^{\alpha \sigma} h_{\sigma \nu, \mu \alpha}-h^{\alpha \sigma}{ }_{, \alpha} h_{\mu \nu, \sigma}-h^{\alpha \sigma} h_{\mu \nu, \sigma \alpha} \\
& -\frac{1}{2} h^{\alpha \sigma}{ }_{, \nu} h_{\sigma \alpha, \mu}-h^{\alpha \sigma} h_{\sigma \alpha, \mu \nu}-\frac{1}{2} h_{, \beta} h_{\mu}{ }^{\beta}{ }_{, \nu} \\
& -\frac{1}{2} h_{, \beta} h_{\nu}{ }^{\beta}{ }_{, \mu}+\frac{1}{2} h_{, \beta} h_{\mu \nu}{ }^{\beta}+h_{\beta \nu}{ }^{, \alpha} h_{\mu \alpha}{ }^{, \beta} \\
& -h_{\beta \nu}{ }^{,{ }^{\alpha}} h_{\mu}{ }^{\beta}{ }_{, \alpha} .
\end{aligned}
$$


We can now use Eq. (71) to substitute for the $k_{\alpha \beta}$ terms that appear in Eq. (65), if we pick a gauge for the $k_{\alpha \beta}$ such that

$$
k^{\alpha}{ }_{\mu, \nu \alpha}+k_{\nu, \mu \alpha}^{\alpha}-k_{\mu \nu, \alpha}{ }^{\alpha}-k_{, \mu \nu}=k_{\mu}{ }^{\sigma}, \nu \sigma-k_{\mu \nu, \sigma}{ }^{\sigma} .
$$

This is easily done if we choose the gauge

$$
k_{\nu}^{\alpha}{ }^{\alpha}=k_{, \nu} .
$$

We can now use Eqs. (71) and (73) along with the de Donder gauge conditions and Eqs. (51) and (52) to simplify the Møller pseudotensor (65) into the form:

$$
\begin{aligned}
-8 \pi_{\mathrm{M}} \mathfrak{T}_{\mu}{ }^{\nu}= & \frac{1}{2} h_{, \mu}{ }^{\nu}+\frac{1}{4} h h_{, \mu}{ }^{\nu}-\frac{1}{2} h_{, \mu \beta} h^{\nu \beta}+h^{\alpha \sigma} h_{\sigma}{ }^{\nu}, \mu \alpha \\
& -\frac{1}{2} h^{\alpha \sigma, \nu} h_{\alpha \sigma, \mu}-h^{\alpha \sigma} h_{\alpha \sigma, \mu}{ }^{\nu} .
\end{aligned}
$$

Inserting Eqs. (46)-(48) for $h_{\alpha \beta}$, we obtain ${ }^{8}$

$$
-8 \pi_{\mathrm{M}} \mathfrak{T}_{0}{ }^{j}=-2 \Phi_{, j 0}-8 \Phi_{, j} \Phi_{, 0}-12 \Phi \Phi_{, j 0} .
$$

Expanding this expression and ignoring terms in the same manner as Eq. (61), we plug into the integral

$$
\frac{d M}{d t}=-\oint_{\mathrm{M}} \mathfrak{T}_{0}{ }^{j} n_{j} r^{2} d \Omega,
$$

and arrive at

$$
\frac{d M}{d t}=-\dot{M}-\dot{\mathcal{I}}_{j k} \mathcal{E}_{j k}
$$

where the $\dot{M}$ term comes from the $-2 \Phi_{, j 0}$ term in Eq. (75), which in turn arises from the fact that the Mbller pseudotensor contains a piece linear in the second derivatives of the metric. Equation (77) can then be written as

$$
\frac{d M}{d t}=-\frac{1}{2} \mathcal{E}_{j k} \frac{d \mathcal{I}_{j k}}{d t} .
$$

Note that in the case of the Møller pseudotensor, the perfect differential term that represents the interaction energy vanishes. This serves to support our intuition that the interaction term is simply a mathematical artifact of our choice of energy localization and that the tidal work is in fact uniquely given by Eq. (1). Comparison with Eq. (41) implies that the Møller pseudotensor corresponds to a Newtonian energy localization given by $\alpha=-2$.

\section{E. Calculation of tidal work using the Bergmann conserved quantities}

To further confirm our intuition about the tidal work, we can calculate $d M / d t$ using the Bergmann conserved quantities given by Eq. (32). However, before we do this, we must determine the form of the arbitrary vector field $\xi^{\sigma}$, on which the momentum density $D^{\mu}$ depends. This form must be such that the integral (34) gives

$$
P^{0}=\varepsilon M+O\left(\varepsilon^{2}\right),
$$

the mass-energy $M$ plus terms of order $\varepsilon^{2}$ which arise from the fact that the surface of integration $\partial \mathcal{V}$ lies in the buffer zone, where spacetime is only locally asymptotically flat.

Computing $P^{0}$ via Eq. (34) first involves calculating the von Freud superpotential (17) in terms of the metric expansion given by Eq. (42). After a rather laborious computation, we find the von Freud superpotential in general gauge and to quadratic order in the metric perturbation:

$$
\begin{aligned}
& -16 \pi_{\mathrm{F}} U_{\alpha}^{[\beta \gamma]}=\varepsilon\left\{\left(\delta_{\alpha}{ }^{\beta} h^{, \gamma}-\delta_{\alpha}{ }^{\gamma}{ }^{, \beta}\right)+\left(\delta_{\alpha}{ }^{\gamma} h_{, \lambda}^{\beta \lambda}-\delta_{\alpha}{ }^{\beta} h^{\gamma \lambda}{ }_{, \lambda}\right)+\left(h_{\alpha}{ }^{\gamma, \beta}-h_{\alpha}{ }^{\beta, \gamma}\right)\right\} \\
& +\varepsilon^{2}\left\{\frac{1}{2} h\left(\delta_{\alpha}{ }^{\beta} h^{, \gamma}-\delta_{\alpha}{ }^{\gamma} h^{, \beta}\right)+\frac{1}{2} h\left(\delta_{\alpha}{ }^{\gamma}{ }^{\beta \lambda}{ }_{, \lambda}-\delta_{\alpha}{ }^{\beta} h_{, \lambda}^{\gamma \lambda}\right)+\frac{1}{2} h\left(h_{\alpha}{ }^{\gamma, \beta}-h_{\alpha}{ }^{\beta, \gamma}\right)+\left(h^{\gamma \lambda} h^{\beta}{ }_{\alpha, \lambda}-h^{\beta \lambda} h^{\gamma}{ }_{\alpha, \lambda}\right)\right. \\
& +h_{, \lambda}\left(\delta_{\alpha}^{\gamma} h^{\beta \lambda}-\delta_{\alpha}{ }^{\beta} h^{\gamma \lambda}\right)+h_{\rho}^{\lambda}\left(\delta_{\alpha}{ }^{\beta} h_{, \lambda}^{\gamma \rho}-\delta_{\alpha}{ }^{\gamma} h_{, \lambda}^{\beta \rho}\right)+h_{\rho, \lambda}^{\lambda}\left(\delta_{\alpha}{ }^{\beta} h^{\gamma \rho}-\delta_{\alpha}^{\gamma} h^{\beta \rho}\right)+\left(h^{\beta \rho} h_{\alpha \rho}{ }^{, \gamma}-h^{\gamma \rho} h_{\alpha \rho}{ }^{, \beta}\right) \\
& \left.+h_{\kappa \rho}\left(\delta_{\alpha}^{\gamma} h^{\kappa \rho, \beta}-\delta_{\alpha}{ }^{\beta} h^{\kappa \rho, \gamma}\right)+\left(\delta_{\alpha}{ }^{\beta} k^{, \gamma}-\delta_{\alpha}{ }^{\gamma}{ }^{, \beta}\right)+\left(\delta_{\alpha}{ }^{\gamma} k_{, \lambda}^{\beta \lambda}-\delta_{\alpha}{ }^{\beta} k_{, \lambda}^{\gamma \lambda}\right)+\left(k_{\alpha}{ }^{\gamma, \beta}-k_{\alpha}{ }^{\beta, \gamma}\right)\right\} \text {. }
\end{aligned}
$$

As in the case of the Møller pseudotensor, we have terms that are linear in the derivatives of the $k_{\alpha \beta}$. For our calculation of $d M / d t$ below, we will find that these terms may again be expressed in terms of the $h_{\alpha \beta}$ by the same choice of gauge as we used in the previous section, Eq. (73). However, we cannot do this for our present calculation of $P^{0}$. Fortunately, this will not be a problem since we only wish to show that $P^{0}$ reduces to the mass-energy $M$ plus terms of order $\varepsilon^{2}$. Since $k_{\alpha \beta}$ is of order $\varepsilon^{2}$ it is not necessary to include the terms containing $k_{\alpha \beta}$ in our calculation of the surface integral in Eq. (34). This is also true of the terms in Eq. (80) that are products of $h_{\alpha \beta}$ and its derivatives. We therefore only need concern ourselves with the first six terms in Eq. (80) (those linear in $h_{\alpha \beta}$ and its derivatives) as only these terms could conceivably affect $P^{0}$ at order $\varepsilon$.

Now, let us make a guess as to the form of the vector field $\xi^{\sigma}$, on which $D^{\mu}$ depends. A volume integral of Bergmann's $D^{0}$ [or the equivalent surface integral of Eq. (34)] will reduce to Eq. (79) only if the form of $\xi^{\sigma}$ is properly constrained. If our spacetime were precisely asymptotically flat, we would expect $\xi^{\sigma}$ to be asymptotically the timelike Killing vector field, 
$\xi^{\sigma} \rightarrow \partial / \partial t+O(1 / r)$. Since our spacetime is not asymptotically flat but only locally asymptotically flat, it is reasonable to expect that $\xi^{\sigma}$ is locally asymptotically Killing, by which we mean that it can be written as the sum of a timelike vector, $\delta_{0}{ }^{\sigma}$, plus deviations $\zeta^{\sigma}$, from this timelike vector that are due to the fact that the spacetime is not flat:

$$
\xi^{\sigma}=\delta_{0}{ }^{\sigma}+\zeta^{\sigma}
$$

As for the form of $\zeta^{\sigma}$, we can construct a quantity which is the most general vector field that is (a) dimensionally correct and (b) constructed only out of the parameters that characterize our spacetime in the slow-motion approximation: $M, \mathcal{I}_{j k}, \mathcal{E}_{j k}$ and their first time derivatives. Such a vector field has the following form (to order $\varepsilon$ ):

$$
\begin{aligned}
& \zeta^{0}=a \dot{M}+b \frac{M}{r}+c \frac{\mathcal{I}_{a b} x_{a} x_{b}}{r^{5}}+d \frac{\dot{\mathcal{I}}_{a b} x_{a} x_{b}}{r^{4}}+e \mathcal{E}_{a b} x_{a} x_{b}+f \dot{\mathcal{E}}_{a b} x_{a} x_{b} r, \\
& \zeta^{k}=g \frac{M x_{k}}{r^{2}}+h \frac{\dot{M} x_{k}}{r^{2}}+i \frac{\mathcal{I}_{a b} x_{a} x_{b} x_{k}}{r^{6}}+j \frac{\dot{\mathcal{I}}_{a b} x_{a} x_{b} x_{k}}{r^{5}}+k \frac{\mathcal{I}_{a k} x_{a}}{r^{4}}+l \frac{\dot{\mathcal{I}}_{a k} x_{a}}{r^{3}}+m \frac{\mathcal{E}_{a b} x_{a} x_{b} x_{k}}{r}+n \dot{\mathcal{E}}_{a b} x_{a} x_{b} x_{k}+o \mathcal{E}_{a k} x_{a} r+p \dot{\mathcal{E}}_{a k} x_{a} r^{2},
\end{aligned}
$$

where the coefficients $a$ through $p$ are real-valued constants.

Using this prescription, we are now able to evaluate $P^{0}$. Our result reduces to Eq. (79) as required. We thus see that the surface integral (34) does indeed give the mass as the only $O(\varepsilon)$ term, plus terms of $O\left(\varepsilon^{2}\right)$ that arise from the facts that our spacetime is locally asymptotically flat and that we evaluate $P^{0}$ on the 2 -surface $\partial \mathcal{V}$ that lies at some finite $r$ in the buffer zone.

Now we wish to compute the tidal work, $d P^{0} / d t$ or $d M / d t$. Taking a time derivative of Eq. (33) and applying Gauss' law, we easily arrive at the expression

$$
\frac{d M}{d t}=-\oint\left(\xi_{\mathrm{F}}^{\sigma} U_{\sigma}^{[j \nu]}\right)_{, \nu} d^{2} S_{j} .
$$

Plugging Eq. (81) into Eq. (84) allows us to write

$$
\frac{d M}{d t}=-\oint_{\mathrm{F}} U_{0}^{[j \nu]}{ }_{, \nu} d^{2} S_{j}-\oint\left(\zeta^{\sigma}{ }_{\mathrm{F}} U_{\sigma}^{[j \nu]}\right)_{, \nu} d^{2} S_{j} .
$$

Let us now think carefully about what terms we actually need to calculate in these integrals. If we examine the first integral in Eq. (85) we realize [comparing with Eq. (80)] that all of the terms in the integrand are of order $\varepsilon^{2}$ and can therefore contribute to the tidal work. However, we again have the problem that we do not know the explicit form of $k_{\alpha \beta}$. Fortunately, if we expand the terms in $\left({ }_{\mathrm{F}} U_{\sigma}^{[\mu \nu]}\right)_{, \nu}$ that depend on $k_{\alpha \beta}$ and apply the gauge condition of Eq. (73), we find that these terms reduce to

$$
\left(k_{\sigma, \nu}^{\nu}{ }^{\mu}-k_{\sigma, \nu}^{\mu}{ }^{\nu}\right) .
$$

Comparison with Eq. (72) shows that this is the same situation encountered with the Møller pseudotensor. We can thus use Eq. (71) to express the first integrand in Eq. (85) entirely in terms of $h_{\alpha \beta}$ and its derivatives, just as we did in the previous section. Applying de Donder gauge and a fair amount of algebraic manipulation we arrive at

$$
\begin{aligned}
-16 \pi_{\mathrm{F}} U_{\sigma}^{[\mu \nu]}{ }_{, \nu}= & \frac{1}{2} h_{, \lambda} h_{, \sigma}^{\mu \lambda}-\frac{1}{4} h_{, \sigma} h^{, \mu}+\delta_{\sigma}{ }^{\mu} h^{\lambda \rho, \nu} h_{\rho \nu, \lambda} \\
& -h_{\lambda \rho, \sigma} h^{\mu \rho, \lambda}+\frac{1}{2} h^{\lambda \rho, \mu} h_{\lambda \rho, \sigma} \\
& -\delta_{\sigma}{ }^{\mu} h^{\kappa \rho, \nu} h_{\kappa \rho, \nu} .
\end{aligned}
$$

Evaluating the components we need for the first integral in Eq. (85), we find ${ }^{8}$

$$
{ }_{\mathrm{F}} U_{0}^{[j \nu]}{ }_{, \nu}=-\frac{1}{4 \pi} \Phi_{, 0} \Phi_{, j}
$$

If we expand this expression as we did in Eq. (61), keeping only terms that can possibly contribute to the tidal work (the products of $\dot{\mathcal{I}} \mathcal{E}$ and $\mathcal{I} \dot{\mathcal{E}}$ ), and evaluate the first integral in Eq. (85), we have

$$
-\oint_{\mathrm{F}} U_{0}^{[j \nu]}{ }_{, \nu} d^{2} S_{j}=-\frac{1}{2} \mathcal{E}_{j k} \frac{d \mathcal{I}_{j k}}{d t}+\frac{3}{10} \frac{d}{d t}\left(\mathcal{I}_{j k} \mathcal{E}_{j k}\right) .
$$

One should note that this is precisely what we obtained using the Einstein pseudotensor [Eq. (63)], and indeed, the lefthand side of Eq. (89) is the surface integral that one would obtain by substituting Eq. (16) into Eq. (58).

We must now evaluate the second integral in Eq. (85) to see if it will contribute to the tidal work. The computation of this integral is made much simpler if we realize that $\zeta^{\sigma}$ is linear in $\varepsilon$. Since the tidal work is quadratic in $\varepsilon$ we only have to concern ourselves with the piece of ${ }_{\mathrm{F}} U_{\alpha}^{[\beta \gamma]}$ that is linear in $\varepsilon$. Applying de Donder gauge to the linear piece of Eq. (80) we can write the second integral in Eq. (85) as 


$$
\begin{aligned}
-\oint\left(\zeta_{\mathrm{F}}^{\sigma} U_{\sigma}^{[j \nu]}\right)_{, \nu} d^{2} S_{j}= & \frac{1}{16 \pi} \oint \frac{1}{2} \zeta^{j}{ }_{, \nu} h^{, \nu}-\frac{1}{2} \zeta^{\nu}{ }_{, \nu} h^{, j} \\
& +\zeta^{\sigma}{ }_{, \nu}\left(h_{\sigma}{ }^{\nu, j}-h_{\sigma}{ }^{j, \nu}\right) d^{2} S_{j} \\
& +O\left(\varepsilon^{3}\right)
\end{aligned}
$$

where we have applied Eqs. (51) and (52). A few pages of algebra, and again ignoring terms that cannot contribute to the tidal work, reduces this to

$$
-\oint\left(\zeta_{\mathrm{F}}^{\sigma} U_{\sigma}^{[j \nu]}\right)_{, \nu} d^{2} S_{j}=\left(\frac{2}{15} c+\frac{3}{5} e\right) \frac{d}{d t}\left(\mathcal{I}_{j k} \mathcal{E}_{j k}\right) .
$$

Combining Eqs. (89) and (91) we finally arrive at an expression for $d M / d t$ in terms of the products that can contribute to the tidal work $(\dot{\mathcal{I}} \mathcal{E}$ and $\mathcal{I} \dot{\mathcal{E}})$ :

$$
\frac{d M}{d t}=-\frac{1}{2} \mathcal{E}_{j k} \frac{d \mathcal{I}_{j k}}{d t}+\left(\frac{3}{10}+\frac{2}{15} c+\frac{3}{5} e\right) \frac{d}{d t}\left(\mathcal{I}_{j k} \mathcal{E}_{j k}\right) .
$$

We can now see conclusively that changing the energy localization has no effect on the tidal work term and merely changes the coefficient in front of the arbitrary interactionenergy term.

\section{CONCLUSIONS}

This paper completes a demonstration that the tidal work caused by the interaction of an isolated body's quadrupole moment $\mathcal{I}_{j k}$ with the electric-type tidal field $\mathcal{E}_{j k}$ of an external universe is unambiguous, despite the ambiguity in the definition of the mass $M$ of such a system. Purdue [2] demonstrated that a gauge change does not lead to any ambiguity in the tidal work in general relativity, nor does one's choice of energy localization in Newtonian theory. We have shown that this energy localization invariance carries over into the general relativistic description. In addition, the work of Booth and Creighton [18], carried out simultaneous with our own, supports the conclusions of Purdue and ourselves through an independent, though equivalent, approach using quasilocal energy techniques.

One of the main motivations for this paper was to strengthen the arguments used by Thorne [4] in his analysis of the stability of neutron stars against radial collapse induced by an external tidal field. The motivation for Thorne's paper was to refute the claims of Wilson, Mathews, and Marronetti [24] regarding the "star-crushing" effect seen in their numerical simulations of binary neutron stars. We believe that our analysis has strengthened Thorne's arguments. Ironically, Thorne and I [25] now have reason, based on current quadrupole tidal coupling, to support the "star-crushing" effects observed by Wilson and Mathews [26] in their revised simulations.

This analysis has only been concerned with the tidal work involving a mass quadrupole moment interacting with an electric-type, quadrupolar tidal field in the slow motion approximation. It seems quite likely that this energy localization and gauge invariance of tidal work can be extended to higher order multipolar couplings. We will leave the demonstration of this for the future.

\section{ACKNOWLEDGMENTS}

I thank Kip Thorne for suggesting this research project and for many insightful discussions as to its solution and the prose of this paper. I also acknowledge Patricia Purdue for useful discussions related to her paper. This research was supported by Caltech, by NSF grant AST-9731698, and NASA grant NAG5 6840.
[1] K. S. Thorne and J. B. Hartle, Phys. Rev. D 31, 1815 (1985).

[2] P. Purdue, Phys. Rev. D 60, 104054 (1999).

[3] X.-H. Zhang, Phys. Rev. D 31, 3130 (1985).

[4] K. S. Thorne, Phys. Rev. D 58, 124031 (1998).

[5] B. Mashhoon, “Absorption of Gravitational Radiation,” GRG Abstracts, Jena Meeting (1980), 188-189.

[6] C. Møller, Ann. Phys. (N.Y.) 4, 347 (1958).

[7] P. G. Bergmann, Phys. Rev. 112, 287 (1958).

[8] C. W. Misner, K. S. Thorne, and J. A. Wheeler, Gravitation (Freeman, San Francisco, 1973), Chap. 20. Referred to in the text as MTW.

[9] L. D. Landau and E. M. Lifshitz, The Classical Theory of Fields, revised fourth English edition, Sec. 96 (ButterworthHeinemann, Oxford, 1998). Referred to in the text as LL.

[10] K. S. Thorne and S. J. Kovács, Astrophys. J. 200, 245 (1975).

[11] E. Nahmad-Achar and B. F. Schutz, Gen. Relativ. Gravit. 19, 655 (1987).

[12] H. Goldstein, Classical Mechanics (Addison-Wesley, Reading, MA, 1980), Chap. 12.
[13] P. A. M. Dirac, General Theory of Relativity (Wiley, New York, 1975).

[14] P. von Freud, Ann. Math. 40, 417 (1939).

[15] J. L. Anderson, Principles of Relativity Physics (Academic, New York, 1967), Chap. 13.

[16] A. Komar, Phys. Rev. 113, 934 (1959).

[17] C.-C. Chang, J. M. Nester, and C.-M. Chen, Phys. Rev. Lett. 83, 1897 (1999).

[18] I. Booth and J. Creighton, Phys. Rev. D 62, 067503 (2000).

[19] J. D. Brown and J. W. York, Jr., Phys. Rev. D 47, 1407 (1993).

[20] C. Cattani and M. De Maria, in The Attraction of Gravitation: New Studies in the History of General Relativity, edited by J. Earman, M. Janssen, and J. D. Norton (Birkhäuser, Boston, 1993), p. 63.

[21] S. V. Babak and L. P. Grishchuk, Phys. Rev. D 61, 024038 (2000).

[22] K. S. Thorne, Rev. Mod. Phys. 52, 299 (1980).

[23] P. Musgrave, D. Pollney, and K. Lake, GRTEnSORII, 1994, Queen's University, Kingston, Ontario, Canada. 
[24] J. R. Wilson, G. J. Mathews, and P. Marronetti, Phys. Rev. D 54, 1317 (1996); J. R. Wilson and G. J. Mathews, Phys. Rev. Lett. 75, 4161 (1995); G. J. Mathews and J. R. Wilson, Astrophys. J. 482, 929 (1997); G. J. Mathews, P. Marronetti, and J. R. Wilson, Phys. Rev. D 58, 043003 (1998); E. Flanagan,
Phys. Rev. Lett. 82, 1354 (1999).

[25] M. Favata and K. S. Thorne (in preparation).

[26] G. J. Mathews and J. R. Wilson, Phys. Rev. D 61, 127304 (2000). 